\title{
Depression Scores and Quality of Life of Vertiginous Patients, Suffering from Different Vestibular Disorders
}

\section{András Molnár ( $\square$ andrasm94@gmail.com )}

Semmelweis University https://orcid.org/0000-0002-4417-5166

\section{Stefani Maihoub}

Semmelweis University

\section{Panayiota Mavrogeni}

Tóth llona Egészségügyi Szolgálat

\section{László Tamás}

Semmelweis University

Ágnes Szirmai

Semmelweis University

\section{Research}

Keywords: Vertiginous patients, depression, quality of life, Dizziness Handicap Inventory, Beck Depression Inventory, Symptom Checklist 90-Revised

Posted Date: November 8th, 2021

DOI: https://doi.org/10.21203/rs.3.rs-1016880/v1

License: (9) (1) This work is licensed under a Creative Commons Attribution 4.0 International License. Read Full License

Version of Record: A version of this preprint was published at European Archives of Oto-RhinoLaryngology on April 18th, 2022. See the published version at https://doi.org/10.1007/s00405-02207366-y. 


\section{Abstract}

\section{Background:}

Vertigo complaints are strongly associated with deterioration of patients' quality of life (QoL). Vertigo and dizziness can result in depression and anxiety; therefore, the diagnosis and management of these concomitant psychiatric disorders can improve the patients' quality of life. The aim of the present study was to contrast the QoL impairment and depression scores of patients suffering from different vestibular disorders.

\section{Methods:}

301 patients were examined due to vertiginous complaints at the Neurotologic Tertiary Rereferral Centre of the Department of Otolaryngology and Head and Neck Surgery of Semmelweis University. These patients completed the Hungarian version of the Dizziness Handicap Inventory (DHI), the Beck Depression Inventory, and the Symptom-Checklist-90-Revised (SCL-90-R) questionnaires.

\section{Results:}

According to neurotologic examination, the distribution of the different diagnoses was as follows: Ménière's disease $(n=101)$, central vestibular disorders $(n=67)$, benign paroxysmal positional vertigo $(n=$ $47)$, vestibular neuritis $(n=39)$, other unilateral peripheral vestibulopathy $(n=18)$, Persistent PosturalPerceptual Dizziness $(n=16)$, vestibular migraine $(n=8)$, and vestibular Schwannoma $(n=5)$. The results of the DHI questionnaire have indicated worsened QoL in $86.4 \%$, out of which $33.6 \%$ was defined as severe. The Beck scale has shown depressive symptoms in $42.3 \%$ of the cases, with severe symptoms in $6.3 \%$. Significantly higher total DHI and Beck scale results were observed in patients with central vestibular disorders, vestibular migraine, Persistent Postural-Perceptual Dizziness and unilateral peripheral vestibulopathy, contrasted to the results of the other four diagnosis groups. The onset of the symptoms did not significantly affect the severity of QoL worsening and depression symptoms.

\section{Conclusion:}

In this study, the QoL of vertiginous patients was worse in general, with the occurrence of depression symptoms. Difference was observed in case of the values of patients with different vestibular disorders, indicating the importance of different factors, e.g., central vestibular compensation, behavioural strategies and psychological factors.

\section{Introduction}

Vertigo is a common complaint, with a 17-30\% prevalence, according to population-based studies [1]. At a neurotological centre, benign paroxysmal positional vertigo (BPPV), Ménière's disease (MD), and vestibular neuritis (VN) can be mentioned as the most frequent diagnoses [2]. Although, other disorders, just like vestibular migraine (VM) [3], Persistent Postural-Perceptual Dizziness (PPPD), and other 
vestibulopathies can also influence the patients' QoL and be associated with psychiatric comorbidities. Psychiatric comorbidities are present in around $30-50 \%$ of the patients suffering from dizziness or vertigo. Among them, one of the most common ones were defined as depression [5], with a prevalence of $4-62 \%$, based on the literature [6]. Due to vertigo or dizziness, impaired QoL often occurs. Psychiatric comorbidities may further worsen the vertiginous patients' QoL. Moreover, due to the QoL impairment and reduced daily activities, inability to work, patients can present psychological distress [7]. Previous studies have observed difference of the QoL impairment and psychiatric comorbidities of patients suffering from different vestibular disorders [8, 9]. Therefore, besides the analysis of patients QoL and depression scores, the present study aimed to analyze the QoL and depression scales in different disorders, resulting in vertigo or dizziness.

\section{Material And Methods Participants}

In this prospective investigation, 301 patients were enrolled. The basic demographical data of the subjects, along with the results of the Dizziness Handicap Inventory (DHI) and Beck depression (BDI) questionnaires, are summarized in Table 1. These patients underwent a complete neurotological examination and have filled out the following questionnaires. The distribution of the different diagnoses, according to neurotologic examination was as follows: $M D(n=101)$, central vestibular disorders $(n=67)$, $\operatorname{BPPV}(n=47), V N(n=39)$, other unilateral peripheral vestibulopathy $(n=18), \operatorname{PPPD}(n=16), \operatorname{VM}(n=8)$, and vestibular Schwannoma $(n=5)$. The study was approved by Semmelweis University Regional and Institutional Committee of Science and Research Ethics: SE RKEB - 203/2021.

\section{Dizziness Handicap Inventory}

The DHI questionnaire is a commonly used self-administered questionnaire for the assessment of QoL impairment, resulting from vertigo and dizziness, validated for different languages. The questionnaire contains 25 questions, with possible answers of 'yes' (4 points), 'sometimes' ( 2 points) and 'no' (0 points). The questions are divided into physical (11 questions), functional (16 questions) and emotional (10 questions) groups. The scoring of the results is done in the following way: 0-14 points indicate a normal handicap, 16-34 mild, 36-52 moderate, while a score over 54 points severe QoL worsening. All of the subjects have filled out a Hungarian version of the DHI [10,11].

\section{Beck Depression Inventory}

To analyze the severity of the depressive symptoms, the shortened, 13-item version of BDI was applied, which was previously reported as a reliable questionnaire for a Hungarian sample [12]. The patients can give answers from' 0 ' ('not at all') to ' 3 ' ('very much'), with a maximal score of 39. 0-5 means no depressive symptoms, scores of 6-11 indicate mild, 12-15 indicate moderately severe, and 16 and above indicate severe depression [13].

\section{Symptom-Checklist-90-Revised}


The Symptom-Checklist-90-Revised (SCL-90-R) questionnaire can examine a broad range of symptoms and psychopathologies, including besides questions regarding depressive symptoms, e.g., for somatization, hostility, phobic anxiety, paranoid ideations, etc. The questionnaire included 90 questions, for each, the patient can give answers from ' 0 ' ('not at all') to ' 4 ' ('extremely'). The subjects who were enrolled in this investigation have completed a Hungarian version of the questionnaire, and the scores of the depressive symptoms (10 questions) were analyzed [14].

\section{Statistical analysis}

IBM SPSS V24 software was used for data processing. Shapiro-Wilk test have indicated that our data was not normally distributed, therefore, Mann-Whitney $U$ test was used to detect for statistically significant difference. The significance level was set up as $p<0.05$. To illustrate the results, boxplot diagrams were included. To analyze correlation, simple linear correlation and Spearman correlation tests were applied. A p value under 0.01 has indicated a significant correlation.

\section{Results}

Table 1. shows the basic demographical data of the patients, along with the summarization of the DHI and $\mathrm{BDI}$ results. 
Table 1

Basic demographical data, $\mathrm{BDI}$ and $\mathrm{DHI}$ results.

\begin{tabular}{|ll|}
\hline Age (mean \pm SD years) & $56.1 \pm 13.4$ \\
\hline Gender (male/female) & $82 / 219$ \\
\hline Duration of the symptoms (vertigo or dizziness; mean \pm SD months) & $93.2 \pm 76$ \\
\hline DHI, $\mathbf{n}($ mean \pm SD) & \\
\hline normal handicap (0-14) & $41(7.5 \pm 4.1)$ \\
\hline mild handicap (16-34) & $90(23.3 \pm 5.2)$ \\
\hline moderate handicap (36-52) & $69(42.9 \pm 4.5)$ \\
\hline severe handicap (54<) & $101(71.5 \pm 9.3)$ \\
\hline Functional score (mean \pm SD) & $17.43 \pm 9.5$ \\
\hline Physical score (mean \pm SD) & $10.5 \pm 5.1$ \\
\hline Emotional score (mean \pm SD) & $13.74 \pm 8.4$ \\
\hline BDI, $n$ (mean \pm SD) & \\
\hline normal (0-5) & $172(2.3 \pm 1.3)$ \\
\hline mild (6-11) & $\mathbf{8 5}(7.8 \pm 1.5)$ \\
\hline moderately severe (12-15) & $25(12.3 \pm 2)$ \\
\hline severe (15<) & $19(21 \pm 2)$ \\
\hline
\end{tabular}

As shown in Table 1., the investigated patients were predominantly females, mostly in their fifties. The total DHI score indicated impaired QoL in $86.4 \%$ of the patients, with severe QoL worsening in $33.6 \%$. The highest mean values were detected in the functional DHI subscore, followed by emotional scores. According to the results of $\mathrm{BDI}$, depression was present in $42.3 \%$, of which was in the severe range in around $6.3 \%$ of the patients.

To analyze whether the duration of the different disorders, i.e., the time from the onset of the symptoms (i.e., vertigo or dizziness) up to the completion of the questionnaire have influence on the scores or not, dot curves were drawn, and correlation tests were applied.

As shown in Fig. 1., no linear correlation was observed between the duration of the disorders and the total $\mathrm{BDI}$ and $\mathrm{DHI}$ scores, either. The Spearman test did not indicate correlation neither in case of $\mathrm{DHI}$ (rho $=0.05, p=0.468)$, nor in case of $B D I(r h o=0.030, p=0.658)$ scores, thus, non-linear correlation was not observed either. Therefore, it can be concluded that the duration of the symptoms has no significant influence on QoL and the occurrence of depressive symptoms. 
The distribution of the total DHI scores of the patients suffering from the different disorders are shown in Fig. 2.

As shown in Fig. 2., lower values of total DHI scores were detected in case of patients suffering from BPPV, MD, VN, and vestibular Schwannoma (Group 1). In case of Schwannoma patients, all DHI scores were in the normal range, indicating no QoL worsening. In terms of central vestibular disorders, VM, PPPD and unilateral peripheral vestibulopathy (Group 2), higher total DHI values were observed, and about $50 \%$ of the scores were found in the severe range. When statistical analysis was carried out using the MannWhitney $U$ test, significant difference was detected between Groups 1 and 2 ( $z$-score: -4.23, $p<0.0001$ ), indicating significantly higher values in Group 2 (i.e., central vestibular disorders, VM, PPPD and unilateral peripheral vestibulopathy). Therefore, patients suffering from the aforementioned disorders have reported significantly worse QoL.

Figure 3. Boxplot showing the distribution of the total BDI values. Black lines in the boxes: median values, box: the middle $50 \%$ of the data, whiskers: upper and lower $25 \%$. (a): normal range (0-5 points), (b): severe range (16+) of the BDI scores. 1: BPPV, 2: MD, 3: VN, 4: vestibular Schwannoma, 5: central vestibular disorders, 6: VM, 7: PPPD, 8: unilateral peripheral vestibulopathy.

As shown in Fig. 3., for the total BDI scores, roughly the same distribution was detected as in case of the total DHI scores. Higher BDI values were observed in Group 2, indicating higher BDI scores of patients with central vestibular disorders, VM and PPPD. Only patients suffering from unilateral vestibulopathy presented lower BDI values, than for total DHI. According to statistical analysis, which was conducted using the Mann-Whitney $\mathrm{U}$ test, the difference between the two groups was statistically significant in this case as well ( $z$-score: $-4.33, p<0.00001)$. This result indicates that besides the higher $\mathrm{DHI}$ values of this group the severity of depressive symptoms was more expressed as well.

The same analysis was conducted for the depression scores according to the SCL-90-R questionnaire. The results are shown in Fig. 4.

Figure 4. shows that higher depression scores based on the SCL-90-R questionnaire were observed in terms of central vestibular disorders, VM, PPPD and unilateral peripheral vestibulopathy. When the values were contrasted using the Mann-Whitney $\mathrm{U}$ test, a statistically significant difference was detected ( $\mathrm{z}$ score: $-3.93, p=0.00008$ ). This result also indicates the similar scores and depression severity, based on $\mathrm{BDI}$ and SCL-90-R, respectively.

\section{Discussion}

In the present study, a great number of subjects suffering from vertigo were involved. The different diagnoses made it possible to analyze the differences between the several groups as well. This is of great importance, as a wide range of vertigo disorders is presented at a neurotologic centre. According to the results of the $\mathrm{DHI}$ questionnaires, $86.4 \%$ of the patients have reported impaired QoL, and significantly higher DHI values were detected in patients suffering from central vestibular disorders, VM, PPPD, and 
unilateral peripheral vestibulopathy, compared to the results of those suffering from the other disorders. BDI have indicated depression symptoms in $42.3 \%$ of the patients, with nearly the same distribution as observed with $\mathrm{DHI}$ regarding the diagnoses. Previous investigations have also contrasted the aforementioned values in different disorders. For instance, Goto et al. have concluded that depressive disorders were more prevalent in patients suffering from sudden deafness and migraine-associated dizziness [8]. According to another investigation, patients suffering from central vestibular disorders have reported the highest $\mathrm{DHI}$ values, and patients with primer functional dizziness presented significantly higher DHI values than those with peripheral vestibular disorders [15]. In the present study, the BDI values of those suffering from PPPD and central vestibular disorders were also significantly higher, than the values of the other diagnosis groups. Zhu et al. have concluded that the emotional, physical, and functional aspects of vertigo had more significant effects on patients with VM than those with BPPV. According to their analysis, DHI scores significantly correlated with depression and anxiety scales [9]. In the present investigation, VM patients also reported significantly higher $\mathrm{DHI}$ and $\mathrm{BDI}$ values contrasted to other vestibulopathies, including BPPV, which is in concordance with the results of the aforementioned study. The results of Möhwald et al. indicated significantly higher DHI values in patients with acute peripheral vestibular disorders than with central ones [16]. This contradicts our results, which have shown significantly lower $\mathrm{DHI}$ and BDI values of most peripheral vestibular disorders, compared to central ones. Although, in their study, patients with acute vestibular symptoms examined at the Emergency Department were enrolled, while most of our patients were not in the acute phase of the disorders, but were investigated on an appointment basis at a tertiary referral centre. However, our analyses found no correlation between the onset of the symptoms, and therefore the duration of the disorders and $\mathrm{DHI}$ and $B D I$ values. MD was defined as the most frequent diagnosis, and is known as a chronic, progressive disorder presenting with episodic vertigo. A meta-analysis regarding MD found that about $50 \%$ of the patients suffer from depression [17]. QoL worsening in MD patients caused by the disorder was also reported $[18,19]$. Moreover, a study concluded that vertigo complaints were the most intrusive symptoms, surpassing other MD symptoms, just like tinnitus or hearing loss. Also, vertigo was most strongly associated with depression and DHI scores [18]. Previous studies showed increased prevalence of affective disorders in episodic vertigo disorders, compared to vestibulopathies with non-episodic vertigo complaints [20]. In the present investigation, patients with episodic (i.e., MD, BPPV, VM) and acute or chronic persistent (i.e., central vestibular disorders, PPPD, VN, unilateral peripheral vestibulopathy) symptoms were also enrolled, but higher $\mathrm{DHI}$ and $\mathrm{BDI}$ values were found in the non-episodic vertigo syndromes group. This is in contrast with previous findings, and highlights the complexity of the background of the QoL impairment and psychiatric symptoms. According to previous findings, $\mathrm{VN}$ also significantly affects the patients' QoL; a moderate impact was reported [21].

The differences in the DHI and BDI values amongst the different disorders cannot be explained only by the vestibular deficit caused by them. As previously reported, there is no correlation between the severity of the vestibular hypofunction, based on objective vestibular testing and QoL and depressive or anxiety symptoms. A study has concluded that the symptom severity and DHI values were not correlated with the results of clinical vestibular tests, but corresponded with several psychological factors (i.e., depression, 
anxiety, cognitive and behavioural responses etc.) [22]. Another study observed no correlation between the DHI scores and high- and low-frequency vestibulo-ocular reflex deficits, respectively. Patients suffering from central vestibular disorders were found to report the highest DHI values, and functional dizziness showed also higher DHI values, contrasted to the parameters of those suffering from peripheral vestibular disorders. As a final conclusion it was stated that more than $96.5 \%$ of the DHI score variances resulted from unaccounted factors, just like central compensation or neurocognitive behavioural factors [15]. Central compensation is a highly complex and vital process after vestibular deficits [23]. Central vestibular compensation involves several anatomical structures of the central nervous system (i.e., vestibular nuclei, vestibulocerebellum, midbrain, dorsolateral/anterior thalamic nuclei, and posterior inferior vestibular cortex) [24], and is influenced by other factors, including behavioural strategies, sociocultural background, age, physical activity, etc. Vertigo or dizziness influence the everyday tasks and can result is psychosocial consequences. Moreover, somatization, i.e., the increased attention to the symptoms, can also be responsible for worsened handicap [25]. Individual coping strategies have also been suspected to be responsible for differences between vestibular tests and the handicap of the patients [26]. The factors mentioned above can influence the central compensation, and therefore, on the QoL and occurrence of psychiatric comorbidities, independently from the objective results of the vestibular tests. Hence, although, questionnaires of QoL and psychiatric symptoms are not specific or sensitive for any vestibular disorders, and therefore, they cannot replace vestibular testing, they still can provide important information regarding the functional and psychological difficulties of vertiginous patients.

\section{Conclusion}

The results of the present research observed significantly impaired QoL in patients with different vertigo disorders, along with the co-occurrence of psychiatric symptoms, presenting themselves as depression. The $\mathrm{DHI}$ and $\mathrm{BDI}$ scores varied amongst the different diagnosis groups, indicating a complex background of the presented symptoms after vestibular damage. Therefore, besides the objective vestibular testing, the self-administered questionnaires may also have an important role.

\section{Abbreviations}

\section{BDI}

Beck Depression Inventory

BPPV

benign paroxysmal positional vertigo

DHI

Dizziness Handicap Inventory

MD

Ménière's disease

PPPD 
Persistent Postural-Perceptual Dizziness

QoL

Quality of life

SCL-90-R

Symptom-Checklist-90-Revised

SD

standard deviation

VN

vestibular neuritis

\section{Declarations}

Acknowledgements This study was supported by EFOP-3.6.3-VEKOP-16-2017-00009 Project and by the ÚNKP-20-4-I New National Excellence Program of The Ministry for Innovation and Technology from the Source of The National Research, Development and Innovation Fund.

Competing interests All of the authors declare that they have no conflict of interest.

Ethical approval All procedures performed in studies involving human participants were in accordance with the ethical standards of the institutional and/or national research committee and with the 1964 Helsinki declaration and its later amendments or comparable ethical standards. The study was approved by Semmelweis University Regional and Institutional Committee of Science and Research Ethics: SE RKEB - 203/2021.

Informed consent Informed consent was obtained from all individual participants included in the study.

Consent for publication Not applicable. Availability of data and materials The datasets generated and/or analysed during the current study are not publicly available due to data protection but are available from the corresponding author on reasonable request.

Funding No funding was received.

Author's contributions: AM: examined the patients, analyzed the questionnaires, performed the statistical analyses and wrote the article. SM: examined the patients, analyzed the questionnaires, and was a major contributor in writing the manuscript. PM: analyzed the questionnaires, critical review of the article. TL: critical review of the article, head of the department. ÁSZ: main tutor of the study, examined the patients, critical review of the article.

\section{References}

1. Cao Z, Zhu C, Zhou Y, Wang Y, Chen M, Ju Y, Zhao X. Risk factors related balance disorder for patients with dizziness/vertigo. BMC Neurol. 2021;21:186. doi: 10.1186/s12883-021-02188-7. 
2. Seidel DU, Park JJ, Sesterhenn AM, et al. Demographic data and seasonal variation in peripheral vestibular disorders in ENT practices in Germany. J Vestib Res. 2019;29:181-190. doi: 10.3233/VES190668.

3. Zaleski-King A, Monfared A. Vestibular Migraine and Its Comorbidities. Otolaryngol Clin North Am. 2021:S0030-6665(21)00115-8. doi: 10.1016/j.otc.2021.05.014.

4. Wang A, Fleischman KM, Kawai K, Corcoran M, Brodsky JR. Persistent Postural-Perceptual Dizziness in Children and Adolescents. Otol Neurotol. 2021 Jun 4. doi: 10.1097/MA0.0000000000003212. doi: 10.1097/MAO.0000000000003212.

5. Herdman D, Sharma H, Simpson A, Murdin L. Integrating mental and physical health assessment in a neuro-otology clinic: feasibility, acceptability, associations and prevalence of common mental health disorders. Clin Med (Lond). 2020;20:61-66. doi: 10.7861/clinmed.2019-0156.

6. Beh SC. The Neuropsychology of Dizziness and Related Disorders. Otolaryngol Clin North Am. 2021:S0030-6665(21)00117-1. doi: 10.1016/j.otc.2021.05.016.

7. Ketola S, Havia M, Appelberg B, Kentala E. Psychiatric symptoms in vertiginous patients. Nord J Psychiatry. 2015;69:287-291. doi: 10.3109/08039488.2014.972976.

8. Lahmann C, Henningsen P, Brandt T, Strupp M, Jahn K, Dieterich M, Eckhardt-Henn A, Feuerecker R, Dinkel A, Schmid G. Psychiatric comorbidity and psychosocial impairment among patients with vertigo and dizziness. J Neurol Neurosurg Psychiatry. 2015;86:302-308. doi: 10.1136/jnnp-2014307601.

9. Goto F, Sugaya N, Arai M, Masuda K. Psychiatric disorders in patients with intractable dizziness in the department of otolaryngology. Acta Otolaryngol. 2018;138:646-647. doi: 10.1080/00016489.2018.1429652.

10. Zhu C, Li Y, Ju Y, Zhao X. Dizziness handicap and anxiety depression among patients with benign paroxysmal positional vertigo and vestibular migraine. Medicine (Baltimore). 2020;99(52):e23752. doi: 10.1097/MD.0000000000023752.

11. Jacobson GP, Newman CW. The development of the Dizziness Handicap Inventory. Arch Otolaryngol Head Neck Surg. 1990;116:424-427. doi: 10.1001/archotol.1990.01870040046011.

12. Mutlu B, Serbetcioglu B. Discussion of the dizziness handicap inventory. J Vestib Res 2013;23:271277. doi: $10.3233 /$ VES-130488.

13. Rózsa S, Szádóczky E, Füredi J. A Beck depresszió kérdőív rövidített változatának jellemzői hazai mintán [Psychometric properties of the Hungarian version of the shortened Beck Depression Inventory]. Psychiatria Hungarica. 2001;16: 384-402.

14. Chibnall JT, Tait RC. The short form of the Beck Depression Inventory: validity issues with chronic pain patients. Clin J Pain. 1994;10:261-266. doi: 10.1097/00002508-199412000-00004

15. Prinz $\mathrm{U}$, Nutzinger DO, Schulz $\mathrm{H}$, et al. Comparative psychometric analyses of the SCL-90-R and its short versions in patients with affective disorders. BMC Psychiatry. 2013;13:104. doi: 10.1186/1471244X-13-104. 
16. Möhwald K, Hadzhikolev H, Bardins S, Becker-Bense S, Brandt T, Grill E, Jahn K, Dieterich M, Zwergal A. Health-related quality of life and functional impairment in acute vestibular disorders. Eur $\mathrm{J}$ Neurol. 2020;27:2089-2098. doi: 10.1111/ene.14318.

17. Patel JJ, Levy DA, Nguyen SA, Rizk HG, Meyer TA. Depression in Ménière's disease: a systematic review and meta-analysis. J Laryngol Otol. 2020;134:293-301. doi: 10.1017/S002221512000081X.

18. Arroll M, Dancey CP, Attree EA, Smith S, James T. People with symptoms of Ménière's disease: the relationship between illness intrusiveness, illness uncertainty, dizziness handicap, and depression. Otol Neurotol. 2012;33:816-823. doi: 10.1097/MA0.0b013e3182536ac6.

19. Anderson JP, Harris JP. Impact of Ménière's disease on quality of life. Otol Neurotol. 2001;22:888894. doi: 10.1097/00129492-200111000-00030.

20. Decker J, Limburg K, Henningsen P, Lahmann C, Brandt T, Dieterich M. Intact vestibular function is relevant for anxiety related to vertigo. J Neurol. 2019;266:89-92. doi: 10.1007/s00415-019-09351-8.

21. Viciana D, Lopez-Escamez JA. Vestibular evoked myogenic potentials and health-related quality of life in patients with vestibular neuritis. Otol Neurotol. 2010;31:954-958. doi: 10.1097/mao.0b013e3181e8fb32.

22. Herdman D, Norton S, Pavlou M, Murdin L, Moss-Morris R. Vestibular deficits and psychological factors correlating to dizziness handicap and symptom severity. J Psychosom Res. 2020;132:109969. doi: 10.1016/j.jpsychores.2020.109969.

23. Sharma KG, Gupta AK. Efficacy and Comparison of Vestibular Rehabilitation Exercises on Quality of Life in Patients with Vestibular Disorders. Indian J Otolaryngol Head Neck Surg. 2020;72:474-479. doi: 10.1007/s12070-020-01920-y.

24. Grosch M, Lindner M, Bartenstein P, Brandt T, Dieterich M, Ziegler S, Zwergal A. Dynamic whole-brain metabolic connectivity during vestibular compensation in the rat. Neuroimage. 2021;226:117588. doi: 10.1016/j.neuroimage.2020.117588.

25. Best C, Eckhardt-Henn A, Diener G, Bense S, Breuer P, Dieterich M. Interaction of somatoform and vestibular disorders. J Neurol Neurosurg Psychiatry. 2006;77:658-664. doi:

10.1136/jnnp.2005.072934.

26. Hallam RS, Stephens SD. Vestibular disorder and emotional distress. J Psychosom Res. 1985;29:407-13. doi: 10.1016/0022-3999(85)90026-1.

\section{Figures}




\section{Correlation between the duration of the symptoms and DHI, BDI scores}
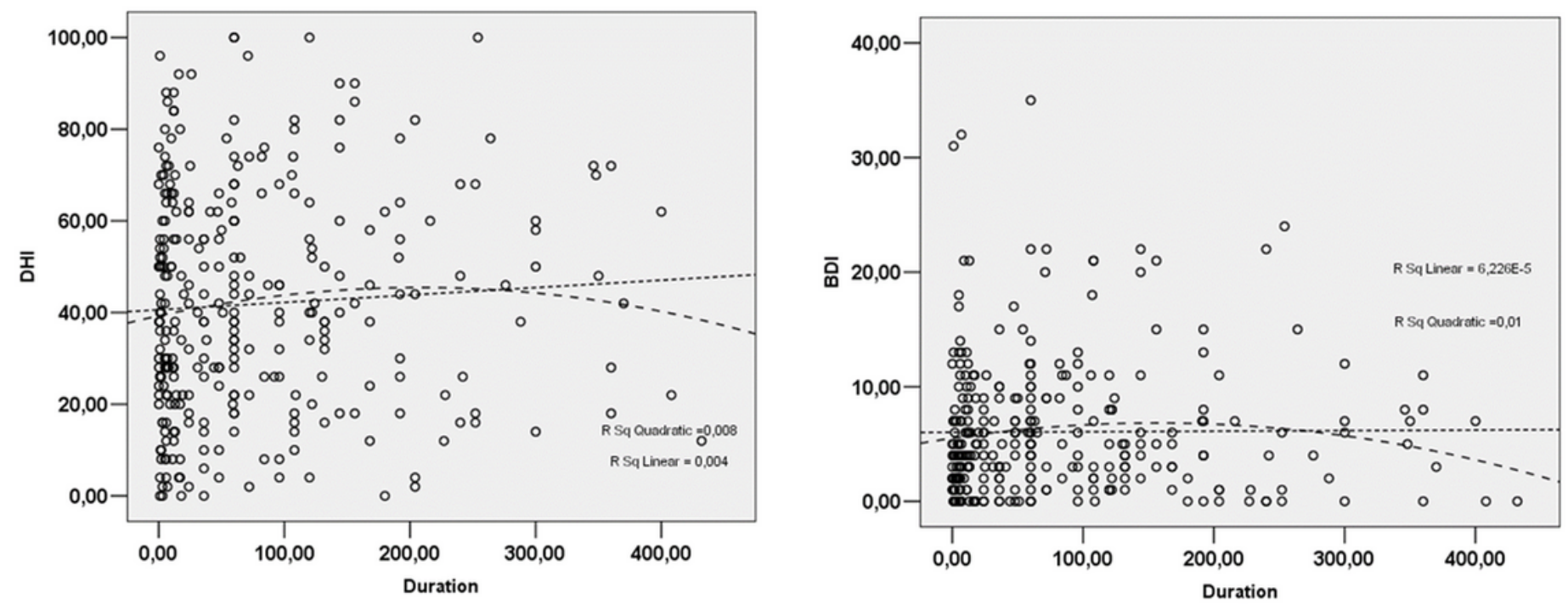

Figure 1

Correlation between the time since the onset of the symptoms (i.e., vertigo or dizziness) and the scores of the questionnaires. 


\section{Total DHI scores according to the different diagnoses}

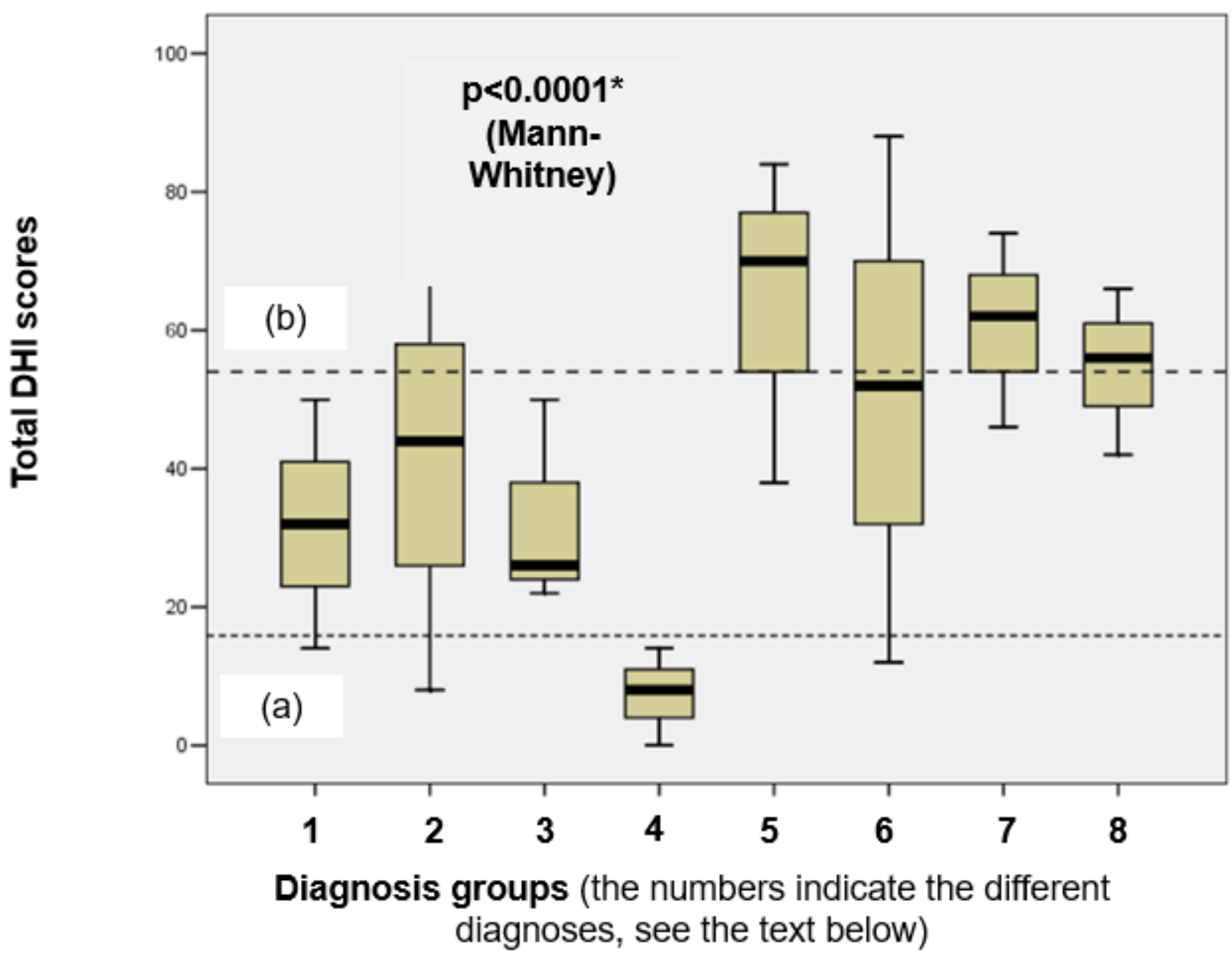

Figure 2

Boxplot showing the distribution of the total DHI values. Black lines in the boxes: median values, box: the middle $50 \%$ of the data, whiskers: upper and lower $25 \%$. (a): normal range (0-14 points), (b): severe range (54+) of the total DHI scores. 1: BPPV, 2: MD, 3: VN, 4: vestibular Schwannoma, 5: central vestibular disorders, 6: VM, 7: PPPD, 8: unilateral peripheral vestibulopathy. 


\section{Total BDI scores according to the different diagnoses}

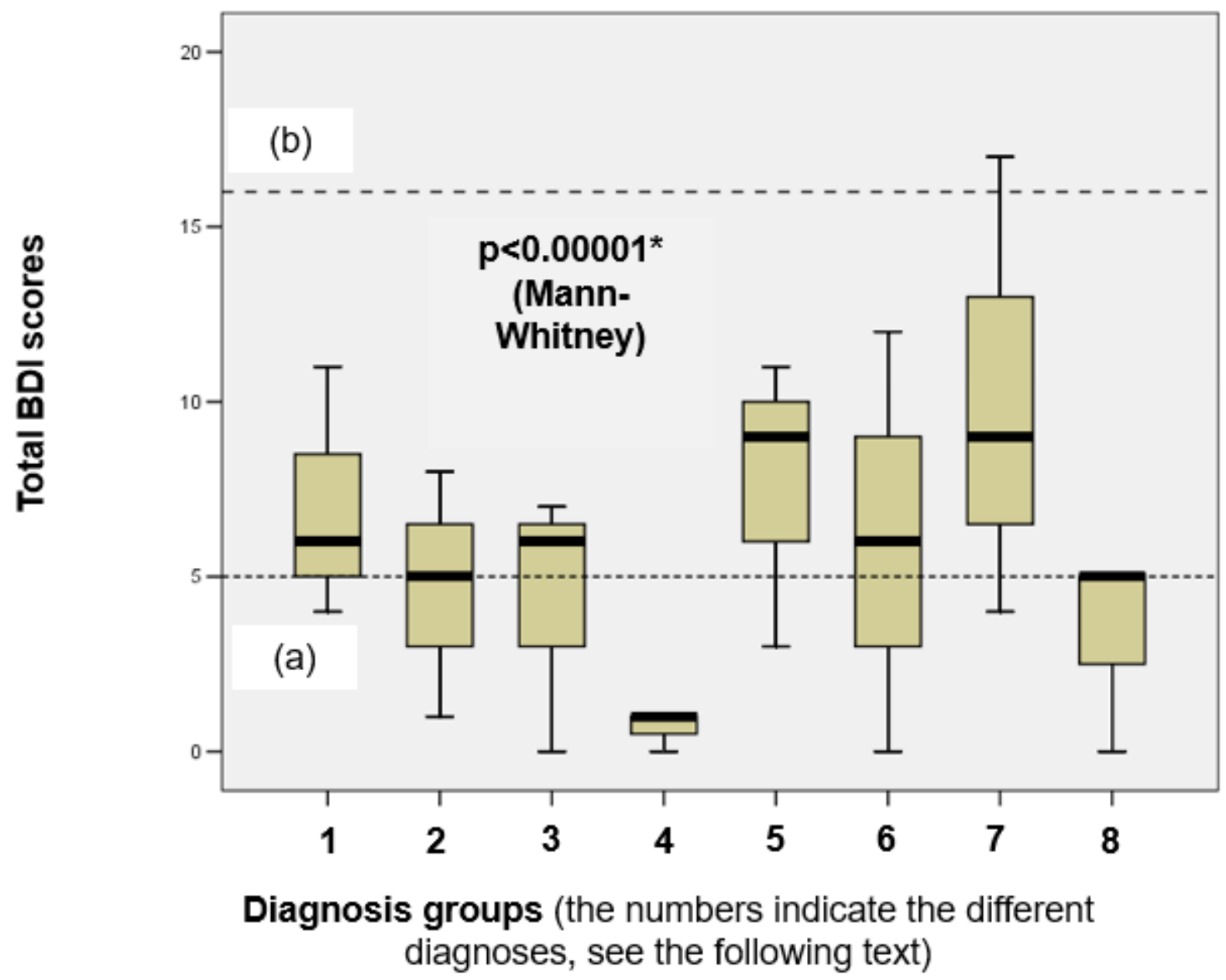

Figure 3

Boxplot showing the distribution of the total BDI values. Black lines in the boxes: median values, box: the middle $50 \%$ of the data, whiskers: upper and lower $25 \%$. (a): normal range (0-5 points), (b): severe range $(16+)$ of the BDI scores. 1: BPPV, 2: MD, 3: VN, 4: vestibular Schwannoma, 5: central vestibular disorders, 6: VM, 7: PPPD, 8: unilateral peripheral vestibulopathy. 